\title{
PASTA UNTABLE DE PAVO ENRIQUECIDA CON FIBRAS, VITAMINAS Y MINERALES ANTIOXIDANTES COMO INGREDIENTES FUNCIONALES PARA EL ADULTO MAYOR
}

\author{
TURKEY SPREAD WITH FIBER, ANTI-OXIDANT \\ VITAMINS AND MINERALS AS FUNCTIONAL \\ INGREDIENTS FOR ELDERLY PEOPLE
}

\author{
Fernando Garrido B. (1), Stephany González Z. (2), \\ Marta Dondero C. (1), Emma Wittig de Penna R. (3)
}

(1) Escuela de Alimentos, Pontificia Universidad Católica de Valparaíso, Valparaíso, Chile.

(2) Ingeniero de Alimentos, Pontificia Universidad Católica de Valparaíso, Chile.

(3) Facultad de Ciencias Químicas y Farmacéuticas. Universidad de Chile, Chile.

\begin{abstract}
A spread for the elderly with turkey, fibers (inulin and oligofructose), anti-oxidant vitamins (A and E) and minerals (selenium and zinc) has been developed. The methodology of Taguchi was used for the optimization of the formulation, considering control factors such as pig butter, hydrated inulin, lactonal, salt, oregano/pepper, garlic and ice. Experimental designs $L 8(27)$ and $L 9(34)$ were used, and sensorial assessments were made. The optimized formulation obtained a value of 4.51 (greater than "good"). The low fat and calorie levels as well as the smooth and pleasant texture stand out in the product. Furthermore, it has been shown that the product is apt and safe for consumption. The spread maintained an optimal sensorial and sanitary quality during storage for 28 days under refrigeration. Elderly showed a $90 \%$ of acceptance and a high preference by the spread. Therefore, the methodology of Taguchi optimized the spread, making it a good alternative of food for the elderly.
\end{abstract}

Key words: Taguchi, anti-oxidants, fiber, elderly, turkey.

Este trabajo fue recibido el 16 de Junio de 2010 y aceptado para ser publicado el 20 de Agosto de 2010.

\section{INTRODUCCIÓN}

El mercado de ingredientes y alimentos funcionales ha experimentado un marcado crecimiento los últimos años debido al aumento de conocimiento por parte de la población en el área de alimentos saludables y la promoción de un estilo de vida sano (1). Por ejemplo, la aparición de mensajes saludables en las etiquetas de los alimentos ha permitido explicar a la población los efectos positivos de la gamma de ingredientes funcionales promoviendo así el consumo de ellos, creando conciencia en los consumidores de alimentarse para nutrirse (2).

Por otro lado, los consumidores esperan que además de los efectos saludables y nutricionales que aporten estos alimentos, sean apetitosos y atractivos (3). Por este motivo las industrias han tenido que desarrollar, innovar y reformular sus productos a fin de adaptarse a los requerimientos de los consumidores manteniendo la calidad de los productos originales $(1,4)$.

En relación a la población chilena, esta se encuentra en un proceso de transición socio-demográfica, presentando un acelerado crecimiento la población envejecida. Grupo dentro del cual existen restricciones en cuanto al tipo la alimentación, producto de los propios procesos fisiológicos que conlleva el envejecimiento. Por ello se busca desarrollar productos que permitan mantener en todo momento la ilusión y el placer de comer y conseguir mediante este placer una nutrición adecuada y eficaz (5).

Por otra parte, cuando se desarrolla un producto, una de las etapas más importantes corresponde a la optimización del producto. Uno de los métodos estadísticos utilizados para la optimización es la metodología de 
Taguchi, la cual esta aplicada al diseño de experimentos multifactoriales. El método de Taguchi además emplea el uso de arreglos ortogonales, que se representan de la forma: $\mathrm{La}(\mathrm{bC})$, donde $\mathrm{L}=$ Indica que es un arreglo ortogonal, $\mathrm{a}=$ número de corridas experimentales (filas), $\mathrm{b}=$ número de niveles para cada factor, $\mathrm{c}=$ número de factores de control a considerar (columnas) (6). Además, esta metodología plantea un diseño fraccionario saturado, el que permite reducir el número de corridas experimentales a realizar, a diferencia de un diseño factorial completo, el que consume tiempo y recursos disponibles. Por ejemplo, un diseño factorial completo 27, involucra 128 experimentos, mientras que el diseño fraccionario saturado de Taguchi sólo considera 8 experimentos. La tabla 1, muestra un ejemplo de L8(27) (7).

El objetivo general de este trabajo consistió en desarrollar una pasta untable de pavo enriquecida con prebióticos, vitaminas y minerales antioxidantes, como ingredientes funcionales para el adulto mayor, empleando la metodología de Taguchi como método de optimización. En forma adicional se busca obtener un producto que sea microbiológicamente seguro para el consumidor, formular una pasta untable con menor contenido de sodio y grasa, junto con ampliar la gama de alimentos que se incluyen normalmente en la dieta del adulto mayor a través de un producto alternativo más sano.

\section{MATERIALES Y MÉTODOS}

La hipótesis planteada en este estudio considera que "Es factible optimizar mediante la metodología de Taguchi, una pasta untable funcional a base de pavo, con el fin de obtener la aceptación sensorial del adulto mayor".

Se utilizaron como materias primas: trutro corto de pavo, manteca de cerdo, emulsificante (lactonal), sal común, especias (pimienta, orégano y ajo), sal de cura, eritorbato de sodio y complementos nutricionales como fibras solubles (inulina y oligofructosa), vitaminas (A y E) y minerales antioxidantes (selenio y zinc).

La formulación inicial se basó en formulaciones encontradas en bibliografía y los aditivos fueron establecidos de acuerdo a recomendaciones dadas por proveedores. En el caso de las vitaminas A y E se fijó cubrir con un $25 \%$ y $30 \%$ de la Ingesta Diaria Recomendada (IDR) por porción de pasta respectivamente más un $40 \%$ de sobredosificación. Con los minerales (selenio y zinc) se fijó cubrir con un $20 \%$ de la IDR/porción de pasta, más un $15 \%$ de sobredosificación, considerando las recomendaciones del Reglamento Sanitario de los Alimentos (8). Las cantidades de inulina y oligofructosa se establecieron a fin de cubrir alrededor del $3 \%$ de la IDR/porción, considerando el nivel de grasa que se deseaba reemplazar por inulina. Para determinar la influencia de la adición de vitaminas y minerales en el producto se realizó una evaluación sensorial mediante Test Triangular con jueces semientrenados (9).

Se utilizó el diseño fraccionario saturado de Taguchi y factores variables como: manteca de cerdo, inulina, emulsificante (lactonal), sal, orégano/pimienta, ajo y hielo. El premix de vitamina A, vitamina E, selenio, zinc, sal de cura, eritorbato y oligofructosa se consideró un factor constante. La variable respuesta (Y) correspondió a la Calidad Sensorial y se utilizó la característica de calidad "mayor es mejor" (a mayor valor de puntaje sensorial mejor es la calidad del producto) (7). Se trabajó inicialmente con el diseño experimental L8(27), que corresponde a 8 corridas experimentales, con 7 factores de control y 2 niveles de trabajo para cada factor de control, posteriormente, con los factores que

\section{TABLA 1}

Arreglo ortogonal $\mathrm{L}_{8}\left(2^{7}\right)$.

\begin{tabular}{cccccccccc} 
Experiencia & A & B & C & D & E & F & G & Resultado \\
& $\mathbf{1}$ & $\mathbf{2}$ & $\mathbf{3}$ & $\mathbf{4}$ & $\mathbf{5}$ & $\mathbf{6}$ & $\mathbf{7}$ & \\
\hline 1 & 1 & 1 & 1 & 1 & 1 & 1 & 1 & $\mathrm{y} 1$ \\
2 & 1 & 1 & 1 & 2 & 2 & 2 & 2 & $\mathrm{y} 2$ \\
3 & 1 & 2 & 2 & 1 & 1 & 2 & 2 & $\mathrm{y} 3$ \\
5 & 1 & 2 & 2 & 2 & 2 & 1 & 1 & $\mathrm{y} 4$ \\
6 & 2 & 1 & 2 & 1 & 2 & 1 & 2 & $\mathrm{y} 5$ \\
7 & 2 & 1 & 2 & 2 & 1 & 2 & 1 & $\mathrm{y} 6$ \\
8 & 2 & 2 & 1 & 1 & 2 & 2 & 1 & $\mathrm{y} 7$ \\
\end{tabular}


resultaron significativos durante esta etapa, se realizó un segundo diseño experimental del tipo L9(34), es decir 9 corridas experimentales con 4 factores de control y 3 niveles de trabajo para cada factor. En forma paralela a las formulaciones se realizaron evaluaciones sensoriales mediante un Test de Puntaje con jueces semientrenados (10), quienes evaluaron atributos sensoriales como apariencia general, olor, color, sabor, textura, de acuerdo a una escala de puntaje de 1 a 5 , donde 5 corresponde a muy bueno y 1 a muy malo.

Con los datos obtenidos, se determinaron los Valores Delta para cada factor, el cual corresponde a la diferencia de los valores promedio de la calidad sensorial entre el nivel mayor y menor de cada factor. Posteriormente, se realizó un Análisis de Varianza (ANOVA) y a partir de estos resultados se procedió a calcular la Ecuación Esperada de Taguchi (7). La que es obtenida mediante la siguiente ecuación:

$\Psi=\mathrm{T}+[(\mathrm{F} 1-\mathrm{T})+(\mathrm{F} 2-\mathrm{T})+(\mathrm{F} 3-\mathrm{T})+(\mathrm{F} 4-\mathrm{T})+\ldots]$

Donde:

$\Psi=$ Respuesta esperada de Taguchi, que representa el valor de la calidad sensorial optima.

$\mathrm{T}=$ Valor del promedio total de la calidad sensorial, que corresponde al valor promedio del total de los datos de calidad sensorial.

F1, F2, F3, F4, ... = Nivel óptimo de los factores 1, 2, 3, $4, \ldots$ respectivamente, que representa el valor de la calidad sensorial del factor $1,2,3,4, \ldots$ respectivamente, en su nivel óptimo (9).

A fin de comprobar que los resultados pueden reproducirse, se elaboró la formulación de confirmación que fue evaluada sensorialmente, mediante Test de Puntaje por jueces semientrenados (10).

La pasta untable funcional optimizada se analizó física, química, microbiológica y sensorialmente.

- Análisis físicos: Se determinó el pH (peachimetro digital) y la actividad de agua, Aw (equipo AQUAL$\mathrm{AB})$.

- Análisis químicos: Se realizó un análisis proximal que incluyó la determinación de humedad según el "método en estufa de aire" (11); Proteínas de acuerdo al "método Kjeldahl" (11), lípidos según el "método por hidrólisis ácida" (11), cenizas de acuerdo al método de "cenizas totales" (11) y carbohidratos totales (calculado por diferencia). Además se determinó el nivel de nitritos, de acuerdo al "método de Grau y Mirna” (11). La determinación de vitamina A y E se realizó por "cromatografía líquida de alta resolución" (12) y los minerales selenio y zinc por "espectroscopia de emisión atómica vía húmeda" (13).

- Análisis microbiológicos: Se efectuaron análisis de Recuento Aerobios Mesófilos, determinación E. Coli, S. Aureus, Cl. Perfringens y Salmonella (14).

- Análisis sensorial: Se realizó una evaluación sensorial con jueces semientrenados quienes evaluaron la pasta untable funcional optimizada y de acuerdo a la característica de textura le otorgaron un puntaje de 1 a 5 , donde 1 corresponde a: No homogénea, muy granulosa; 2: defectuosa, poco homogénea, granulosa; 3 : no tan homogénea, levemente granulosa; 4 : agradable, suave, homogénea y 5: muy agradable, muy suave/muy homogénea.

Además, se efectuó una evaluación sensorial con 30 adultos mayores (hombres y mujeres) entre 60 y 85 años de edad, quienes evaluaron dos tipos de pastas de pavo sobre galletas: pasta $\mathrm{A}$ (pasta untable funcional) y pasta B (paté de pavo comercial), a fin de determinar el grado de satisfacción producido por el producto, para lo cual se emplearon escalas hedónicas verbales de 5 puntos y en forma paralela se determinó la preferencia de las pastas (10).

Como última etapa, se realizó un estudio de vida útil por un periodo de 28 días, durante el cual la pasta untable funcional optimizada fue almacenada a $5^{\circ} \mathrm{C}$ en un envase flexible (polietileno y nylon) transparente. Para cada tiempo de muestreo (0, 14 y 28 días) se hizo: a) un recuento Aerobios Mesófilos, el que debía cumplir con lo exigido en el Reglamento Sanitario de los Alimentos (8) para ser considerado aceptable y b) una Evaluación sensorial mediante un Test de Puntaje (10) con una escala de 1 a 5 , donde se considera 3 como nota mínima de aceptación.

\section{RESULTADOS}

La tabla 2, resume los niveles utilizados en la optimización de la formulación utilizando la metodología de Taguchi.

Los resultados de la calidad sensorial del diseño experimental L8(27), se presentan en la tabla 3. A continuación, la tabla 4, presenta los resultados de los valores Delta y la tabla 5, el Análisis de Varianza (ANOVA) de los datos obtenidos en las experiencias.

La tabla 6 muestra el resumen de los datos utilizados en el segundo diseño experimental: L9(34). Los resultados de la calidad sensorial del segundo diseño, se presentan en la tabla 7. Mientras que la tabla 8 detalla los resultados de los valores Delta y la tabla 9, muestra el Análisis de Varianza (ANOVA) de los datos obtenidos de esta experiencia.

La Ecuación Esperada de Taguchi ( $\Psi)$, arrojó un 


\section{TABLA 2}

Factores y niveles de trabajo.

Factores de control

A: Manteca de cerdo

B: Inulina hidratada

C: Emulsionante (lactonal)

D: Sal

E: Orégano/pimienta

F: Ajo

G: Hielo

Nivel $1(\%)$

$\begin{array}{cc}9 & 12 \\ 11 & 13 \\ 1 & 1,2 \\ 0,8 & 1 \\ 0,1 / 0,1 & 0,16 / 0,2 \\ 0,05 & 0,11 \\ 24 & 26\end{array}$

$\mathrm{Y}($ respuesta $)=$ Calidad sensorial

\section{TABLA 3}

Respuestas de la calidad sensorial obtenidas en el diseño experimental $L_{8}\left(2^{7}\right)$.

\begin{tabular}{|c|c|c|c|c|c|c|c|c|c|c|}
\hline \multirow{3}{*}{ Experiencia } & \multirow{3}{*}{$\mathbf{A}$} & \multirow{3}{*}{ B } & \multirow{3}{*}{$\mathbf{C}$} & \multirow{3}{*}{ D } & \multirow{3}{*}{$\mathbf{E}$} & \multirow{3}{*}{$\mathbf{F}$} & \multirow{3}{*}{ G } & \multicolumn{3}{|c|}{ Calidad sensorial } \\
\hline & & & & & & & & \multicolumn{2}{|c|}{ Repeticiones } & \multirow[t]{2}{*}{ Promedio } \\
\hline & & & & & & & & $\mathbf{R} 1$ & $\mathbf{R} 2$ & \\
\hline 1 & 1 & 1 & 1 & 1 & 1 & 1 & 1 & 4,05 & 3,96 & 4,00 \\
\hline 2 & 1 & 1 & 1 & 2 & 2 & 2 & 2 & 3,76 & 3,69 & 3,72 \\
\hline 3 & 1 & 2 & 2 & 1 & 1 & 2 & 2 & 4,02 & 3,96 & 3,99 \\
\hline 4 & 1 & 2 & 2 & 2 & 2 & 1 & 1 & 4,15 & 4,06 & 4,10 \\
\hline 5 & 2 & 1 & 2 & 1 & 2 & 1 & 2 & 3,66 & 3,41 & 3,53 \\
\hline 6 & 2 & 1 & 2 & 2 & 1 & 2 & 1 & 4,10 & 4,07 & 4,09 \\
\hline 7 & 2 & 2 & 1 & 1 & 2 & 2 & 1 & 4,13 & 4,03 & 4,08 \\
\hline \multirow[t]{2}{*}{8} & 2 & 2 & 1 & 2 & 1 & 1 & 2 & 3,88 & 3,75 & 3,81 \\
\hline & & & & & & & \multicolumn{3}{|c|}{ Promedio total } & 3,92 \\
\hline
\end{tabular}

\section{TABLA 4}

Valores Delta para el diseño experimental $\mathbf{L}_{8}\left(2^{7}\right)$.

Niveles

\section{Factores}

\begin{tabular}{cccccccc} 
& A & B & C & D & E & F & G \\
\hline 1 & 3,95 & 3,84 & 3,90 & 3,90 & 3,97 & 3,86 & 4,07 \\
2 & 3,88 & 4,00 & 3,93 & 3,93 & 3,86 & 3,97 & 3,76 \\
Delta & 0,07 & 0,16 & 0,03 & 0,03 & 0,11 & 0,11 & 0,31
\end{tabular}


valor de 4,33, considerando un valor del promedio total de la calidad sensorial (T) de 4,06 (tabla 7) y los factores en su nivel óptimo.

$\Psi=\mathrm{T}+[($ Inulina Hidratada en nivel mayor $-\mathrm{T})+$ (Orégano/Pimienta en su nivel menor $-\mathrm{T})+($ Ajo en su nivel mayor $-\mathrm{T})+($ Hielo en su nivel menor $-\mathrm{T})]=4,33$.

Por otra parte, el Test de Puntaje aplicado a la formulación óptima, obtuvo un puntaje promedio de 4,51. Mientras que la tabla 10, resume los resultados obtenidos del análisis físico, químico y microbiológico realizado a la pasta untable funcional optimizada.

En relación a la evaluación sensorial de calidad de textura, la pasta untable funcional optimizada obtuvo un puntaje promedio de 3,45 .

La figura 1, muestra los resultados de la evaluación a los consumidores potenciales. Mientras que en relación al test de preferencia, 25 individuos de un total de 30 mostró preferencia por la pasta untable funcional.
Por otro lado, los resultados del estudio de vida útil se detallan en la tabla 11.

\section{DISCUSIÓN}

Mediante la evaluación de Test Triangular se estableció que el nivel de vitaminas y minerales no fueron percibidos en forma significativa por los panelistas $(\mathrm{p}=0,05)$. Por lo que estos niveles son apropiados para la formulación y se consideraron constantes.

A partir de los resultados de la tabla 4, se demostró que los factores que resultaron significativos y que obtuvieron una mayor respuesta (bajo el criterio "mayor respuesta es mejor") fueron: B2 (inulina hidratada en nivel mayor), E1 (orégano/pimienta en su nivel menor), F2 (ajo en su nivel mayor) y G1 (hielo en su nivel menor).

En la tabla 5, se observa que los factores G, B, E y $F$ fueron los que ejercieron una influencia significativa $(a=0,05)$ en la variable respuesta, por lo que se conside-

\section{TABLA 5}

Análisis de varianza (ANOVA) para el diseño experimental $L_{8}\left(2^{7}\right)$.

$\begin{array}{cccccc}\text { Factores } & \begin{array}{c}\text { Grados de } \\ \text { libertad }\end{array} & \begin{array}{c}\text { Suma de } \\ \text { cuadrados }\end{array} & \begin{array}{c}\text { Cuadrado } \\ \text { medio }\end{array} & \text { Valor F } & \begin{array}{c}\text { Peso } \\ \text { ponderado }(\%)\end{array} \\ \text { A } & 1 & 2,41 * 10-2 & 2,41 * 10-2 & 3,32 & 3,69 \\ \text { B } & 1 & 0,10 & 0,10 & 13,91 & 15,46 \\ \text { C } & 1 & 2,11 * 10-3 & 2,11 * 10-3 & 0,29 & 0,32 \\ \text { D } & 1 & 3,87 * 10-3 & 3,87 * 10-3 & 0,53 & 0,59 \\ \text { E } & 1 & 5,10 * 10-2 & 5,10 * 10-2 & 7,03 & 7,81 \\ \text { F } & 1 & 4,45 * 10-2 & 4,45 * 10-2 & 6,13 & 6,81 \\ \text { G } & 1 & 0,37 & 0,37 & 50,77 & 56,42 \\ \text { Error } & 8 & 5,81 * 10-2 & 7,26 * 10-3 & & 8,89 \\ \text { Total } & 15 & 0,65 & & & 100\end{array}$

\section{TABLA 6}

Factores y niveles de trabajo segundo diseño experimental.

\begin{tabular}{|lccc|}
\hline Factores de control & Nivel 1 $(\boldsymbol{\%})$ & Nivel 2 $(\boldsymbol{\%})$ & Nivel 3 $(\boldsymbol{\%})$ \\
\hline B: Inulina Hidratada & 11 & 12 & 13 \\
E: Orégano/pimienta & $0,1 / 0,1$ & $0,13 / 0,15$ & $0,16 / 0,2$ \\
F: Ajo & 0,05 & 0,08 & 0,11 \\
G: Hielo & 24 & 25 & 26 \\
\hline Y (respuesta) $=$ Calidad sensorial & & &
\end{tabular}


raron en un segundo diseño matricial ortogonal L9(34). Los factores A, C y D presentaron una baja contribución porcentual y no son significativos, por lo que en el nuevo diseño experimental fueron considerados constantes y se trabajó con su nivel más bajo, por un criterio económico.

De acuerdo los resultados expuestos en la tabla 9 , el factores $\mathrm{G}$ y B fueron los que presentaron mayor influencia en la calidad sensorial, a diferencia de los factores $\mathrm{E}$ y $\mathrm{F}$, que evidenciaron una baja contribución porcentual. Por lo tanto, considerando los resultados expuestos en las tablas 8 y tabla 9 los niveles que proporcionaron las mejores respuestas fueron: B3 (Inulina Hidratada en nivel mayor), El (orégano/pimienta en su nivel menor), F3 (ajo en su nivel mayor) y G1 (hielo en su nivel menor).

Del Test de Puntaje realizado a la formulación que resultó óptima, se obtuvo un puntaje promedio de 4,51, superior al valor teórico esperado de acuerdo a la ecuación de Taguchi $(4,33)$, lo que indica que el producto fue optimizado satisfactoriamente.

De los resultados expuestos en la tabla 10, destacó el bajo nivel de lípidos y calorías, en comparación a un paté comercial, ya que fue utilizado un menor porcentaje de grasa (manteca de cerdo) en la formulación, el que fue sustituido por inulina y además se utilizó carne de pavo previamente desgrasada dentro de la formulación. Por otro lado, el valor de carbohidratos totales evidenció un mayor nivel por incluir dentro de su determinación el aporte de fibra proporcionado por la inulina y oligofructosa. El análisis microbiológico y de nitritos, indicó que estos cumplen con lo exigido por el nuevo Reglamento Sanitario de los Alimentos

\section{TABLA 7}

Respuestas de la calidad sensorial obtenidas en el diseño experimental $\mathrm{L}_{9}\left(3^{4}\right)$.

\begin{tabular}{cccccccc} 
Experiencia & B & E & F & G & \multicolumn{4}{c}{ Calidad Sensorial } \\
Repeticiones & Promedio \\
& & & & & & R1 & R2 \\
1 & 1 & 1 & 1 & 1 & 4,06 & 4,19 & 4,12 \\
2 & 1 & 2 & 2 & 2 & 3,92 & 4,13 & 4,03 \\
3 & 1 & 3 & 3 & 3 & 3,70 & 3,92 & 3,81 \\
4 & 2 & 1 & 2 & 3 & 3,86 & 3,82 & 3,84 \\
5 & 2 & 2 & 3 & 1 & 4,18 & 4,21 & 4,20 \\
6 & 2 & 3 & 1 & 2 & 4,00 & 4,09 & 4,04 \\
7 & 3 & 1 & 3 & 2 & 4,29 & 4,31 & 4,30 \\
8 & 3 & 2 & 1 & 3 & 4,04 & 4,02 & 4,03 \\
9 & 3 & 3 & 2 & 1 & 4,16 & 4,16 & 4,16 \\
& & & & & Promedio total & $\mathbf{4 , 0 6}$
\end{tabular}

TABLA 8

Valores Delta para el diseño experimental $\mathrm{L}_{9}\left(3^{4}\right)$.

\section{Niveles}

Nive

Delta
B

3,99

4,03

4,16

0,17

\section{Factores}

E

4,09

4,08

4,00

0,09

\section{G}

4,16

4,12

3,89

0,27 
para cecinas cocidas. Con respecto a los niveles de vitaminas y minerales encontrados en el producto, fueron menores a los incorporados inicialmente al producto, especialmente en el caso de la vitamina E y zinc. Dichas pérdidas se debieron a que durante el procesamiento y almacenamiento del producto las vitaminas son afectadas por factores como presencia de oxígeno, luz, calor y lípidos (15), sumado al hecho que la vitamina E es uno de los primeros antioxidantes que es consumido durante el periodo de iniciación de la oxidación de grasas, ejerciendo su acción de antioxidante durante esa fase (16). Otra posible causa de las pérdidas, pudo deberse a una deficiente homogenización durante la preparación del premix o durante el mezclado manual.

De la evaluación sensorial de calidad de textura, se obtuvo un puntaje de 3,45 , lo que indicó que la pasta es suave, agradable, homogénea y levemente granulosa. Considerando que este tipo de productos son consumidos sobre galletas o pan, se piensa que el defecto de la granulosidad es mínimo y no será percibido por el consumidor.

Por otra parte, de la evaluación a los consumidores potenciales para determinar el grado de satisfacción, el 90\% de ellos señaló agrado por la pasta untable funcional (Pasta A), con una calificación promedio de 4,3, es decir superior a "me gusta". Además la pasta untable presentó una amplia preferencia por el adulto mayor, ya que se consideran como mínimo 21 juicios para establecer diferencias significativas $(\mathrm{p}=0,05)$

En relación al estudio de vida útil (tabla 11), durante el tiempo que se mantuvo el producto en almacenamien-

\section{TABLA 9}

Análisis de varianza (ANOVA) para el diseño experimental $\mathrm{L}_{9}\left(3^{4}\right)$.

\begin{tabular}{cccccc} 
Factores & $\begin{array}{c}\text { Grados de } \\
\text { libertad }\end{array}$ & $\begin{array}{c}\text { Suma de } \\
\text { cuadrados }\end{array}$ & $\begin{array}{c}\text { Cuadrado } \\
\text { medio }\end{array}$ & Valor F & Peso ponderado (\%) \\
\hline B & 2 & 0,10 & $5,13 * 10-2$ & 7,67 & 22,12 \\
E & 2 & $2,69 * 10-2$ & $1,34 * 10-2$ & 2,01 & 5,79 \\
F & 2 & $2,67 * 10-2$ & $1,34 * 10-2$ & 2,00 & 5,76 \\
G & 2 & 0,25 & 0,12 & 18,49 & 53,34 \\
Error & 9 & $6,03 * 10-2$ & $6,70 * 10-3$ & & 12,99 \\
Total & 17 & 0,46 & & & 100
\end{tabular}

\section{TABLA 10}

Resultados análisis físicos, químicos y microbiológicos

\begin{tabular}{lccc}
\hline Análisis físicos & Valor & Análisis microbiológicos & Valor \\
Peso porción $(\mathrm{g})$ & 15 & R. total a $35^{\circ} \mathrm{C}(\mathrm{UFC} / \mathrm{g})$ & 240 \\
$\mathrm{Aw}\left(\mathrm{a} 20^{\circ} \mathrm{C}\right)$ & 0,988 & E. coli $(\mathrm{NMP} / \mathrm{g})$ & $<3$ \\
$\mathrm{pH}\left(\mathrm{a} 20^{\circ} \mathrm{C}\right)$ & 6,58 & S. aureus $(\mathrm{UFC} / \mathrm{g})$ & $<10$ \\
Análisis químicos & Valor & Cerfringens $(\mathrm{UFC} / \mathrm{g})$ & $<10$ \\
Humedad $(\%)$ & 68,68 & Salmonella $25 \mathrm{~g}$ & Ausencia $(-)$ \\
Proteínas $(\mathrm{NT} \times \mathrm{x}$ 6,25) $(\%)$ & 13,94 & & \\
Lípidos $(\%)$ & 7,98 & & \\
Cenizas $(\%)$ & 1,47 & & \\
Carbohidratos totales $(\%)$ & 7,93 & & \\
Calorías $/ 100 \mathrm{~g}$ & 158,26 & & \\
Nitrito $(\mathrm{mg} / \mathrm{kg})$ & 120,14 & & \\
\end{tabular}


to, el nivel de microorganismos se encontró bajo el límite exigido por el Reglamento Sanitario de los Alimentos y con respecto a la calidad sensorial, se observó una disminución en el puntaje promedio a medida que trascurrió el tiempo, siempre sobre 4, por lo que este producto se considera apto y seguro para su consumo.

\section{CONCLUSIONES}

Los factores que más influyeron en la calidad sensorial de la pasta untable fueron: hielo, inulina hidratada y especias (orégano/pimienta y ajo). En relación a las vitaminas y minerales, se detectaron pérdidas en el producto optimizado, específicamente de vitamina E y zinc, indicando que su actividad como antioxidantes seria más baja en el adulto mayor. Se determinó que se cumple con todos los requerimientos microbiológicos y de nitritos estipulados para cecinas cocidas, indicando que es un producto inocuo y apto para el consumo. El estudio de vida útil indicó que la pasta untable funcional optimizada logró mantener una óptima calidad sensorial y sanitaria.

La inulina adicionada a la pasta untable funcional permitió sustituir más de la mitad de la grasa que presenta un paté comercial, logrando obtener una textura suave, homogénea, húmeda y agradable. La pasta untable evidenció ser una buena alternativa de alimento funcional para ser incluida en la dieta del adulto mayor, al presentar

FIGURA 1

Grado de satisfacción hedónica de pastas de pavo.

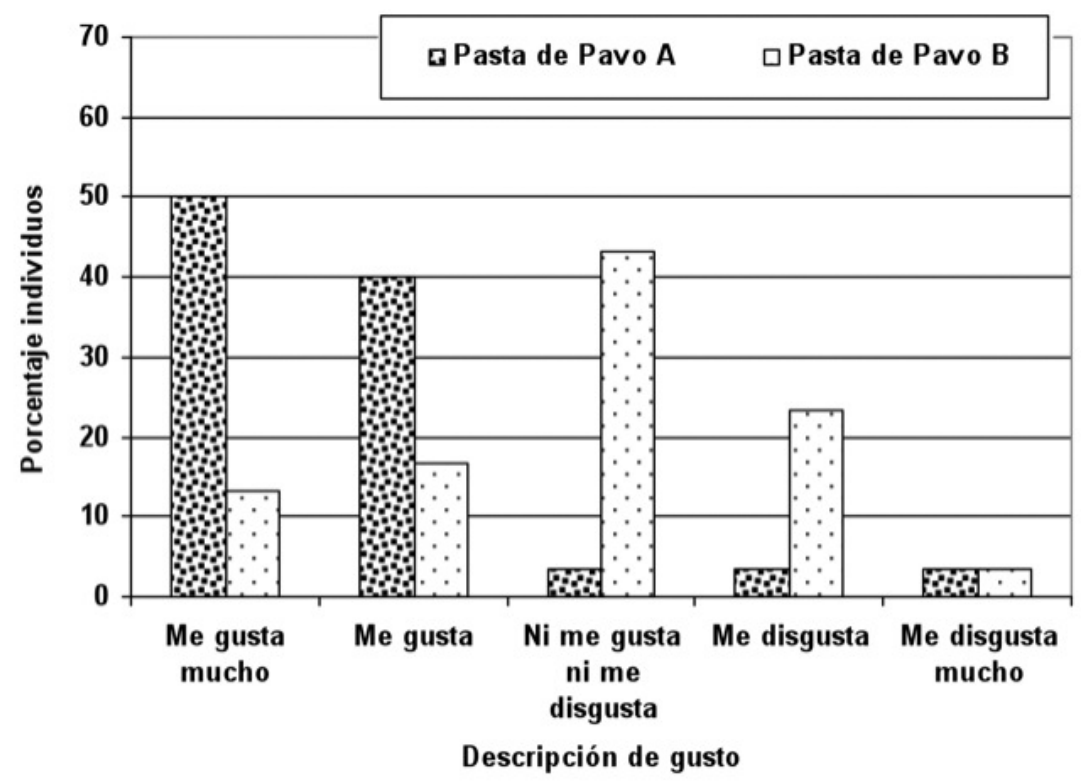

TABLA 11

Resultados del estudio vida útil.

Tiempo

0

14

28
Recuento total a $35^{\circ} \mathrm{C}$ (UFC/g)

\section{Calidad sensorial}

$$
\begin{gathered}
4,51 \\
4,37 \\
4,2
\end{gathered}
$$


un bajo nivel de grasa y sodio en su formulación (lo que equivale a una reducción de un $68 \%$ de grasa y $56 \%$ de sal en relación a un paté comercial).

\section{RESUMEN}

Se desarrolló una pasta untable destinada al adulto mayor con pavo, fibras solubles (inulina y oligofructosa), vitaminas (A y E) y minerales antioxidantes (selenio y zinc). La formulación se optimizó con la metodología de Taguchi, considerando como factores de control: manteca de cerdo, inulina hidratada, emulsionante, sal, orégano/pimienta, ajo y hielo. Se utilizaron los diseños experimentales L8(27) y L9(34) y se realizaron evaluaciones sensoriales, obteniendo la formulación optimizada un puntaje de 4,51 (superior a "bueno"). Del producto destacó el bajo nivel de lípidos y calorías, la textura suave-agradable y se demostró que el producto es apto y seguro para el consumo, manteniendo su calidad sensorial y sanitaria durante 28 días de almacenamiento en refrigeración. El adulto mayor mostró un $90 \%$ de aceptación y alta preferencia por la pasta. Por lo tanto, la metodología de Taguchi logró optimizar la pasta untable, constituyendo una buena alternativa de alimento para el adulto mayor.

Palabras clave: Taguchi, antioxidantes, fibra, adulto mayor, pavo.

Dirigir la correspondencia a:

Profesor

Fernando Garrido B.

Escuela de Alimentos

Pontificia Universidad Católica de Valparaíso

Waddington 716

Valparaíso, Chile

Email:fgarrido@ucv.cl

\section{BIBLIOGRAFÍA}

1. Day L. Seymour R. Pitts K, Konczak I. Lundin L. Incorporation of Functional Ingredients into Food. Trends Food Science Technol. 2008;1-8.

2. Williams P- Ghosh D. Health Claims and Functional Foods. Nutr Dietetic. 2008; 65 (Suppl.3):589-93.

3. Puupponen R. Aura A. Oksman-Caldentey K. Myllärinen P. Saarela M. Mattila-Sandholm T. Poutanen
K. Development of Functional Ingredients for Gut Health. Trends Food Science Technol. 2002; 13:3-11.

4. Fogliano, V. Vitaglione P. Review: Functional Food: Planning and development. Mol Nut Food Res. 2005; 49:256-62.

5. Salvador, G. Bultó, L. Larousse de la Dietética y de la Nutrición. Editorial Larousse. Barcelona. España. 2001.

6. Instituto Tecnológico de Chihuahua. Curso Ingenieríade Calidad_México_[anline]_Disponible. http./f www itchedu.mx/academic/industrial/ingcalidad index.htm

7. ASI Internacional. Centro para el Método de Taguchi. Instituto Tecnológico y de Estudios Superiores de Monterrey. Introducción a la Ingeniería de la Calidad. Manual del Curso. México. 1989.

8. Ministerio de Salud. Nuevo Reglamento Sanitario de los Alimentos. Editorial Publiley. Santiago. Chile. 2004.

9. Wittig de Penna E. Evaluación Sensorial. Una metodología actual para tecnología de alimentos. Santiago. Chile. 1981.

10. Anzaldúa A. La Evaluación Sensorial de los Alimentos en la Teoria y la Práctica. Editorial Acribia S.A. Zaragoza. España. 1994.

11. Instituto de Salud Pública. Manual Métodos Análisis Físico-Químico de Alimentos, Aguas y Suelos. Santiago.Chile. 1998.

12. Driskell W. Neese J. Bryant C. Bashor M. Measurement of vitamin $\mathrm{A}$ and vitamin $\mathrm{E}$ in human serum by high performance liquid chromatography. J Chromatography 1982; 231:439- 44.

13. INN. Aguas residuales - Métodos de análisis - Parte 30: Determinación de selenio - Método de espectrofotometría de absorción atómica por generación continua de hidruros. NCh 2313/30. Of 1999.

14. Instituto de Salud Pública. Manual de Técnicas Microbiológicas Alimentos-Aguas. Santiago.Chile. 1998.

15. Badui S. Química de los Alimentos. Editorial Pearson Educación. México. 1999.

16. Maga, J. Tu, A. Food Additive Toxicology. Editorial Marcel Dekker Inc. New Cork. USA. 1995. 